PPPL-3184 - Preprint: June 1996, UC-420, 427

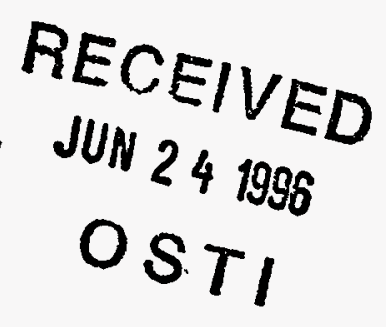

\title{
MODELING OF A-PARTICLE REDISTRIBUTION BY SAWTEETH IN TFTR USING FPPT CODE
}

\author{
N. N. GORELENKOV, R. V. BUDNY, H. H. DUONG, ET AL.
}

Results from recent DT experiments on TFTR to measure the radial density profiles of fast confined well trapped a-particles using the Pellet Charge eXchange (PCX) diagnostic [PETROV M. P., et.al. Nucl. Fusion, 35 (1995) 1437] indicate that sawtooth oscillations produce a significant broadening of the trapped alpha radial density profiles. Conventional models consistent with measured sawtooth effects on passing particles do not provide satisfactory simulations of the trapped particle mixing measured by PCX diagnostic. We propose a different mechanism for fast particle mixing during the sawtooth crash to explain the trapped a-particle density profile broadening after the crash. The model is based on the fast particle orbit averaged toroidal drift in a perturbed helical electric field with an adjustable absolute value. Such a drift of the fast particles results in a change of their energy and a redistribution in phase space. The energy redistribution is shown to obey the diffusion equation, while the redistribution in toroidal momentum $\mathrm{Pj}$ (or in minor radius) is assumed stochastic with large diffusion coefficient and was taken at. The distribution function in a pre-sawtooth plasma and its evolution in a post-sawtooth crash plasma is simulated using the FokkerPlanck Post-TRANSP (FPPT) processor code. It is shown that FPPT calculated a-particle distributions are consistent with TRANSP Monte-Carlo calculations. Comparison of FPPT results with Pellet Charge eXchange (PCX) measurements shows good agreement for both sawtooth free and sawtoothing plasmas.

\section{DISCLAIMER}

\begin{abstract}
This report was prepared as an account of work sponsored by an agency of the United States Government. Neither the United States Government nor any agency thereof, nor any of their employees, makes any warranty, express or implied, or assumes any legal liability or responsibility for the accuracy, completeness, or usefulness of any information, apparatus, product, or process disclosed, or represents that its use would not infringe privately owned rights. Reference herein to any specific commercial product, process, or service by trade name, trademark, manufacturer, or otherwise does not necessarily constitute or imply its endorsement, recommendation, or favoring by the United States Government or any agency thereof. The views and opinions of authors expressed herein do not necessarily state or reflect those of the United States Government or any agency thereof.
\end{abstract}


May 10, 1996

\title{
MODELING OF $\alpha$-PARTICLE REDISTRIBUTION BY SAWTEETH IN TFTR USING FPPT CODE*
}

\author{
N. N. GORELENKOV † R. V. BUDNY, H. H. DUONG; \\ R. K. FISHER ${ }^{\ddagger}$, S. S . MEDLEY and M. P. PETROV \\ Princeton Plasma Physics Labo atory \\ P.O. Box 451, Princeton, NJ 08543-0451
}

\begin{abstract}
Results from recent DT experiments on TFTR to measure the radial density profiles of fast confined well trapped $\alpha$-particles using the Pellet Charge eXchange (PCX) diagnostic [M. P. Petrov, et.al. Nucl. Fusion, 35 (1995) 1437] indicate that sawtooth oscillations produce a significant broadening of the trapped alpha radial density profiles. Conventional models consistent with measured sawtooth effects on passing particles do not provide satisfactory simulations of the trapped particle mixing measured by PCX diagnostic. We propose a different mechanism for fast particle mixing during the sawtooth crash to explain the trapped $\alpha$-particle density profile broadening after the crash. - The model is based on the fast particle orbit averaged toroidal drift in a perturbed helical electric field with an adjustable absolute value. Such a drift of the fast particles results in a change of their energy and a redistribution in phase space. The energy redistribution is shown to obey the diffusion equation, while the redistribution in toroidal momentum $P_{\varphi}$ (or in minor radius) is assumed stochastic with large diffusion coefficient and was taken flat. The distribution function in a pre-sawtooth plasma and its evolution in a post-sawtooth crash plasma is simulated using the Fokker-Planck Post-TRANSP (FPPT) processor code. It is shown that FPPT calculated $\alpha$-particle distributions are consistent with TRANSP Monte-Carlo calculations. Comparison of FPPT results with Pellet Charge eXchange (PCX) measurements shows good agreement for both sawtooth free and sawtoothing plasmas.
\end{abstract}

*This work is supported by US DoE contract DE-AC02-76-CHO-3073 and US DoE Grant DE-FG0392ER54150

$t$ Permanent affiliation: TRINITI, Troitsk, Russia

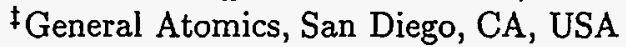

$\S$ Ioffe Physical-Technical Institute, St.Petersburg, Russia 


\section{Introduction}

The physics of confined fast charged fusion products in tokamak plasmas is becoming the reactor relavant issue for experimental study. Trapped $\alpha$-particles are studied in Deuterium-Tritium (DT) TFTR plasma with the PCX diagnostic [1]. During the sawtooth activity in the plasma, trapped particles were seen to be redistributed and their profiles were significantly broadened [2]. Similar effects were seen by the $\alpha$-CHERS (alpha particle CHarge Exchange Recombination Spectroscopy) diagnostic [3]. Trapped particles are easily affected by toroidal magnetic field ripple, which may give rise to energetic ion fluxes to the first wall of tokamak reactor. If ripples are weak these fluxes are not important, provided there is no anomalous radial transport of fast particles. Therefore, sawtooth broadening of alphas profiles may lead to some restrictions for ITER-like tokamaks.

The modeling of the confined fast particle redistribution during the sawtooth crash has started recently [4]. Models were developed mainly for neutral beam injected passing particles, which were assumed to have a small radial deviation from the magnetic surface. Therefore such particles do not differ from the plasma ions and should obey the same equations. Also assumed was the conservation of particle energy during the mixing. Slowing down distribution functions of beam ions that were obtained give good agreement with measurements of neutron fluxes due to beam-plasma fusion reactions.

PCX results indicate, however, that the local density of trapped particles on the outer bypass of the torus was significantly increased after the sawtooth crash, which may be explained only by the radial expulsion of trapped $\alpha$-particles from the center accompanied by nonconservation of their energy. Our goal is to develop a model which can describe the PCX data and has measurable critical parameters. In this paper we assume that the toroidal drift in the perturbed perpendicular electric field determines the energy change of fast particles during the sawtooth crash. We propose a mechanism of fast particle energy redistribution, which is shown to obey the diffusion type equation. A helical electric field is assumed to be generated during the so-called "collapse" period of the sawtooth oscillations on a very short time scale $\tau_{c r} \simeq 10^{-5}-10^{-4} \sec$ (crash time) [5]. This 
approach provides the possibility for fast particles to undergo significant displacement within the mixing radius during the crash. It can be considered as a resonant interaction, even though the mode itself has very low frequency and was assumed not to be rotating during the short crash. Therefore, particles with energy higher than some critical value $\mathcal{E}_{c r}$ perform toroidal precession during the crash and do not interact with perturbed electric field. We introduce $\mathcal{E}_{c r}$ from the comparison of particle toroidal rotation and sawtooth crash time $\tau_{c r}$. It plays the role of an adjustable parameter in the comparison with the experimental data. The comparison is discussed in the paper.

The paper is organized as follows. In Section 2, we consider the physical constraints for fast particle mixing and obtain the equations for energy redistribution. In Section 3 , the FPPT code for fast particle distribution function calculation is described and compared with TRANSP Monte-Carlo calculations and with sawtooth free PCX measurements. Section 4 is devoted to the comparison of the FPPT sawtooth mixing model with experimental data. Summary and conclusions are given in Section 5 .

\section{Fast Particle Redistribution during a Sawtooth Crash.}

\subsection{Physical constraints and characteristic electric fields}

We start the analysis with the formulation of physical constraints for fast particle mixing and introduce three motion invariants, which are the variables to describe the fast particle distribution function in a tokamak. The first variable is the perpendicular adiabatic invariant or magnetic momentum $\mu=\mathcal{E}_{\perp} / B$, where $\mathcal{E}_{\perp}$ is the perpendicular particle energy and $B$ is magnetic field. Magnetic momentum is conserved during the crash time due to the fast cyclotron rotation of the particles

$$
\tau_{c r} \gg 2 \pi \omega_{c}^{-1}
$$

where $\omega_{c}$ is the fast particle cyclotron frequency.

The second variable is the longitudinal invariant or toroidal momentum $P_{\varphi}$

$$
P_{\varphi}=\frac{e_{\alpha} \psi}{m_{\alpha} c}-v_{\|} R
$$


where $\varphi$ is toroidal angle, $e_{\alpha}$ and $m_{\alpha}$ are the charge and mass of $\alpha$-particle, respectively, $\psi$ is the poloidal magnetic flux, $R$ is the major radius of the torus, $v_{\|}=v \sqrt{1-p B / B_{0} R_{0}}$, where the subscript " 0 " means that the value is taken at the tokamak magnetic axis, and

$$
p=\mu B_{0} R_{0} / \mathcal{E}
$$

which is equal to the major radius of the drift orbit bounce point for trapped particles. $P_{\varphi}$ is not conserved during the crash, because of the breakdown of toroidal symmetry. In helical perturbed magnetic fields, the fast particle invariant $P_{\varphi}$ may change due to the stochastization of magnetic field lines in the presence of two or more nonlinearly interacting modes $[6,7]$ and/or freezing of fast particles in the central and peripheral magnetic fluxes during the collapse [4]. The first mechanism leads to rather flat distributions in $P_{\varphi}$, while the second one may result in hollow profiles [8], provided a small radial width of fast particle drift orbits is assumed. In tokamaks such as the Joint European Torus (JET) or Tokamak Fusion Test Reactor (TFTR) [9], the radial excursions of fast particles $\Delta_{b}$ are significant and may be equal to the mixing radius $r_{m}$, which is of the order of inversion radius. Under these conditions the problem needs extensive numerical calculations. For the sake of simplicity, we will assume that the distribution in $P_{\varphi}$ is flat at each moment. This assumption seems reasonable for passing particles as a zero order approximation. Trapped particles do not follow the helically perturbed magnetic lines, and we estimate the diffusion in $P_{\varphi}$ for trapped particles as follows. The radial component of perturbed magnetic field $B_{r}$ is expressed through the island half width $w$ by the familiar equation:

$$
\frac{B_{r}}{B} \simeq \frac{w^{2}}{r^{2}} \frac{\epsilon S m}{4 q}
$$

where $S=r q^{\prime} / q$ is shear, $\epsilon$ is the inverse aspect ratio, $m$ is the poloidal mode number of the perturbation, and the expression must be evaluated at the rational magnetic surface $r=r_{s}$, where $q\left(r_{s}\right)=m / n=1$ for $m=n=1$. The displacement in minor radius during one toroidal precession of the trapped particle is $\Delta r=2 \pi B_{r} q R / B$ which will give the diffusion coefficient, if we assume the stochastization of magnetic field lines, 
$D=(\Delta r)^{2} / \tau_{p r}$, where $\tau_{p r} \simeq 2 \pi r / q v_{d r}$ is the toroidal precession time, and $v_{d r}$ is the drift velocity. Then, the time required for the fast particle to diffuse in $P_{\varphi}$ (or in minor radius) from the center to the periphery is

$$
\Delta t=\frac{r_{s}^{2}}{D} \tau_{p r}
$$

For $\alpha$-particles in a typical TFTR plasma (see Section 4) with $S=10^{-1}$ and $m=1$, Eq.(5) gives $\Delta t=10^{-3} \mathrm{sec}$, which is less than or of the order of the sawtooth precursor evolution time [5].

The third variable may be either particle energy $\mathcal{E}$ or $p$, which are assumed not to be conserved here. Three variables $\mu, P_{\varphi}, \mathcal{E}$ or $\mu, P_{\varphi}, p$ determine the particle orbit in axisymmetric tokamak equilibria. Fast motion along the drift orbit may be ignored because of the inequality $\tau_{b} \ll \tau_{c r}$, where $\tau_{b}$ is the bounce (for trapped) or transit (for passing particles) period of fast drift motion.

Due to the magnetic momentum conservation, the number of particles with given $\mu$ must be conserved:

$$
N=<f_{\alpha}\left(\mu, P_{\varphi}, p\right)>_{P_{\varphi}, p} \equiv \int f_{\alpha}\left(\mu, P_{\varphi}, p\right) \mathcal{J} d P_{\varphi} d p=\text { const }
$$

where $f_{\alpha}\left(\mu, P_{\varphi}, p\right)$ is the $\alpha$-particle distribution function, $\mathcal{J}$ is the Jacobian of the transition from 6 th dimension phase space to variables $\mu, P_{\varphi}, p$ with ignorable toroidal angle, gyrophase rotation and particle position on the drift orbit:

$$
\mathcal{J}=\frac{(2 \pi)^{2} B B_{0} R_{0}}{\omega_{c}} \frac{\tau_{b}}{p^{2}}
$$

To explain the PCX experimental data (see Section 3.3), one must find the mechanism which leads to energy change during the sawtooth crash. The effect of the parallel electric field is too small to change the fast particle energy [10] and is neglected here, while the perpendicular electric field leads to the change of particle energy due to the toroidal drift motion and is propotional to $\left\langle\mathrm{v}_{d r} \cdot \mathbf{E}_{\perp}\right\rangle$, where $\mathrm{V}_{d r}$ is toroidal drift velocity, $\mathbf{E}_{\perp}$ is the electric field in the laboratory frame of reference, and angle brackets mean the orbit averaging. To find the characteristic electric field we assume that the bulk plasma is described by the ideal magnetohydrodynamic (MHD) formalism and has 
helical symmetry during the crash with $m=n=1$ [11] and has the local plasma velocity vector

$$
\mathbf{v}_{E p l} \simeq v_{0}\left[\mathrm{e}_{r} \cos (m \theta-n \varphi-\omega t)-\mathrm{e}_{\theta} \sin (m \theta-n \varphi-\omega t)\right]
$$

Here $v_{0}=r_{m} / \tau_{c r}, \mathbf{e}_{r}$ and $\mathbf{e}_{\theta}$ are unity vectors in the radial and poloidal directions, respectively, and we introduced the frequency of the mode rotation $\omega$. Then, in the laboratory frame of reference the electric field is given by

$$
\mathbf{E}_{\perp}=c^{-1} \mathbf{B} \times \mathbf{v}_{E p l}=E_{0}\left[\mathbf{e}_{r} \sin (m \theta-n \varphi-\omega t)+\mathbf{e}_{\theta} \cos (m \theta-n \varphi-\omega t)\right]
$$

where $E_{0}=r_{m} B / c \tau_{c r}$. Another estimate of the electric field comes from the poloidal component of Faraday's law $\nabla \times \mathbf{E}_{\perp}=-c^{-1} \partial \mathbf{B} / \partial t$, provided $E_{\|}=0$ and we know the change of central safety factor $\Delta q_{0}$. Then, the change of poloidal magnetic field is $\Delta B_{\theta} \simeq B \epsilon \Delta q_{0}$, which together with Faraday's law gives

$$
E_{0} \simeq \frac{q R \Delta B_{\theta}}{c \tau_{c r}\left(m-n q_{0}\right)} \simeq \frac{r_{m} B}{c \tau_{c r}} \frac{\Delta q_{0}}{m-n q_{0}}
$$

which is consistent with our previous estimate Eq.(9). For a crash time of $\tau_{c r} \simeq 3 \times$ $10^{-5} \mathrm{sec}$, we evaluate the perturbed electric potential:

$$
\frac{e \phi}{r_{m}}=e B r_{m} / c \tau_{c r} \simeq 1 \mathrm{keV} \mathrm{cm}^{-1} .
$$

The potential is of the order of plasma temperature within the mixing radius $r_{m} \simeq 40 \mathrm{~cm}$, but may lead to a significant change of fast particle energy, as will be shown.

\subsection{Fast particle energy change in the model electric field}

A single particle changes its energy in the electric field given by Eq.(9) in accordance with the following equation

$$
\frac{d \mathcal{E}}{d t}=e_{\alpha}\left\langle\mathrm{v}_{d r} \cdot \mathrm{E}_{\perp}\right\rangle \simeq-\frac{E_{0}}{B} \frac{c \mathcal{E}}{R}\left\langle\left(2-\frac{p B}{B_{0} R_{0}}\right) \cos ((m-1) \theta-n \varphi-\omega t)\right\rangle,
$$

where the toroidal drift velocity was taken neglecting plasma pressure effect as $\mathrm{v}_{d r}=$ $\mathcal{E}\left(2-p B / B_{0} R_{0}\right)[\mathbf{B} \times \nabla \ln B] / m_{\alpha} \omega_{c} B$. Note that the toroidal angle is no longer ignorable and we assume that before the crash particles were equally distributed in $\varphi$. For 
analytical estimates we will use the approximation of large tokamak aspect ratio with circular magnetic surfaces, and zero radial width of drift particle orbits. Near the $q=1$ surface we can write $\varphi=q \theta+\varphi_{m d}$, where $\varphi_{m d}$ is toroidal angle where fast particles intersect the midplane on the low magnetic field side. Then, with the magnetic field in the form $B=B_{0} R_{0} / R$ and $R=R_{0}+r \cos \theta$, one can obtain the expression for the toroidal precession rate:

$$
\frac{d \varphi_{m d}}{d t} \simeq \frac{q}{e_{\alpha} B} \frac{c \mathcal{E}}{r R}\left\langle\left(2-\frac{p B}{B_{0} R_{0}}\right) \cos \theta\right\rangle .
$$

Comparing Eq.(12) and Eq.(13) and using helical symmetry, which implies $m-n q \ll 1$, we obtain the equation for the energy variation during the sawtooth crash

$$
\frac{d \mathcal{E}}{d t}=-\frac{E_{0} e_{\alpha} r}{q} \cos \left(n \varphi_{m d}-\omega t\right) \frac{d \varphi_{m d}}{d t}
$$

After time integration Eq.(14) results in the fast particle energy change during the crash

$$
\Delta \mathcal{E}=-\frac{2 E_{0} e_{\alpha} r}{\mathcal{R} n q} \sin \left(\mathcal{R} \frac{d \varphi_{m d}}{d t} \frac{n \tau_{c r}}{2}\right) \cos \left(\mathcal{R} \frac{d \varphi_{m d}}{d t} \frac{n \tau_{c r}}{2}+n \varphi_{m d 0}\right)
$$

where we assumed that the precession rate is time independent, $\mathcal{R}=1-\omega\left(n d \varphi_{m d} / d t\right)^{-1}$ is the resonace factor and $\varphi_{m d 0}$ is taken before the crash. In a small orbit width approximation the precession rate is given by the following expression:

$$
\frac{d \varphi_{m d}}{d t} \simeq \frac{q}{e_{\alpha} B} \frac{c \mathcal{E}}{r R} h\left(\kappa^{2}\right) \equiv \frac{2 \pi}{\tau_{p r 0}} h\left(\kappa^{2}\right)
$$

where

$$
h\left(\kappa^{2}\right)=\left(2-\frac{p}{R_{0}}\right)\left\{\begin{array}{ll}
-1+2 E\left(\kappa^{2}\right) / K\left(\kappa^{2}\right), & \text { if } \quad \kappa<1 \\
1-2 \kappa^{2}+2 \kappa^{2} E\left(\kappa^{-2}\right) / K\left(\kappa^{-2}\right), & \kappa>1
\end{array},\right.
$$

$E, K$ are full eliptic integrals of second and first order, respectively, and

$$
\kappa^{2}=(1-\epsilon) \frac{R_{0}(1+\epsilon)-p}{2 \epsilon p}
$$

which is $\kappa<1$ for trapped and $\kappa>1$ for passing particles. In the limit cases of well trapped $(\kappa \rightarrow 0)$ and passing $(\kappa \rightarrow \infty)$ particles we have

$$
h\left(\kappa^{2}\right) \simeq\left\{\begin{array}{lll}
1-\kappa^{2}, & \text { if } \quad \kappa \rightarrow 0 \\
-\kappa^{-2} / 2, & & \kappa \rightarrow \infty
\end{array} .\right.
$$


The displacement of the alternative to the energy variable, the bounce tip position $p$, results from the conservation of $\mu$ :

$$
\begin{aligned}
\Delta p & =-p \frac{\Delta \mathcal{E}}{\mathcal{E}}=\frac{2 p E_{0} e_{\alpha} r}{n \mathcal{R} q \mathcal{E}} \sin \left(\mathcal{R} \frac{d \varphi_{m d}}{d t} \frac{n \tau_{c r}}{2}\right) \cos \left(\mathcal{R} \frac{d \varphi_{m d}}{d t} \frac{n \tau_{c r}}{2}+n \varphi_{m d 0}\right) \\
& \equiv \Delta_{0} \cos \left(\mathcal{R} \frac{d \varphi_{m d}}{d t} \frac{n \tau_{c r}}{2}+n \varphi_{m d 0}\right) .
\end{aligned}
$$

We introduce a useful parameter

$$
\mathcal{E}_{c r}=\frac{\mathcal{E} \tau_{p r 0}}{n \pi \tau_{c r}}=\frac{2 \omega_{c} m_{\alpha} r R}{n \tau_{c r} q}
$$

which is approximately equal to the energy of particles which have the precession period satisfying $\tau_{p r}=\tau_{c r}$. From Eqs. $(16,20)$ we obtain

$$
\frac{\Delta_{0}}{r_{m}}=\frac{p}{R_{0}} \frac{\mathcal{E}_{c r}}{\mathcal{E R}} \sin \left(\frac{h\left(\kappa^{2}\right) \mathcal{E} \mathcal{R}}{\mathcal{E}_{c r}}\right) \text {. }
$$

Figure 1 shows an example of the displacement versus bounce point position $p$ for $\omega=0$ and different particle critical energies $\mathcal{E}_{c r}$. As one can see from Eq.(21), the interaction is resonant, which gives the largest effect for particles with either $\mathcal{E}=0, \omega=0$ or $\mathcal{R}=0$. In the comparison with experiments we will assume $\omega=0$, while the second case with $\mathcal{R}=0$ is analogous. Note, that experimental observations indicate low rotation period in comparison with the crash time.

It follows from the Fig. 1 that low energetic $\left(\tau_{c r}<\tau_{p r}\right)$, well trapped particles have maximum energy change and maximum bounce tip displacement. We can also see from Eq.(19) that the variation of the particle energy or $p$ is an oscillating function of $\varphi_{m d}$ and needs to be averaged over $\varphi_{m d}$ in order to find the distribution function after the crash (see next section).

\subsection{Redistribution in energy or banana tip position $p$}

Let us assume that $p$ displacement is small $\Delta p / R_{0} \ll \epsilon$ and take the fast particle distribution function in $p$ as a $\delta$ function and flat in $\varphi$. It can be easily shown using Eq.(19), that after the averaging over $\varphi$ we obtain the transformation

$$
\delta(p) \rightarrow G(p)=\int \delta(p+\Delta p) \frac{d \varphi}{2 \pi}=\frac{\eta\left(\Delta_{0}^{2}-p^{2}\right)}{\pi \sqrt{\Delta_{0}^{2}-p^{2}}}
$$


where $\eta$ is Heaviside step function. It follows from this equation that the distribution function after the crash $f_{\alpha+}(p)$ is expressed through the one before the crash $f_{\alpha-}(p)$ as:

$$
f_{\alpha+}(p)=\mathcal{J}^{-1} \int f_{\alpha-}\left(p^{\prime}\right) G\left(p-p^{\prime}\right) \mathcal{J} d p^{\prime}=\mathcal{J}^{-1} \int f_{\alpha-}\left(p^{\prime}+p\right) G\left(p^{\prime}\right) \mathcal{J} d p^{\prime}
$$

We expand $f_{\alpha-}$ in series and perfom the integration, which gives

$$
f_{\alpha+}(p)=f_{\alpha-}(p)+\frac{\partial^{2} f_{\alpha-}(p)}{\partial p^{2}} \frac{\Delta_{0}^{2}}{4} .
$$

Noticing that the transformation of the distribution function was made during the time $\tau_{c r}$, we construct the diffusive type equation from Eq.(24) for the $p$-mixing of the fast particle distribution function as follows

$$
\frac{\partial f_{\alpha}}{\partial t}=D_{p} \frac{\partial^{2} f_{\alpha}}{\partial p^{2}}
$$

where the "diffusion" coefficient is $D_{p}=\Delta_{0}^{2} / 4 \tau_{c r}$. Equations $(23,25)$ are valid for small $p$ displaceḿent, while well trapped low energetic particles have $\Delta_{0} \sim r_{m}$. For such a group of particles the transformation formula in Eq.(23) does not conserve particles with given $\mu$, and the postcrash $\alpha$-particle density must be normalized to the precrash value.

We also propose the other nonintegral procedure for $p$ redistribution in contrast to integral Eq.(23). This new procedure can be used for large $p$ displacement, and is based on the assumption of a diffusive nature of the mixing. This means that the distribution function tends to be flattened in $p$ with an exponentially fast rate and the following procedure may be used:

$$
f_{\alpha+}(p)=f_{\alpha-}(p) g+C_{n}(1-g)
$$

where $C_{n}$ does not depend on $p, C_{n}=\left\langle f_{-}(1-g)\right\rangle_{P_{\varphi}, p} /\langle 1-g\rangle_{P_{\varphi}, p}, g$ is flattening rate which must satisfy the following conditions: (1) $0<g<1$; (2) $g \rightarrow 0$ at $\kappa \rightarrow 0, \mathcal{E} \rightarrow 0$ giving full mixing for well trapped particles;(3) $g \rightarrow 1$ at $\mathcal{E} \rightarrow \infty$ representing fast precession drift without changing energy; and, (4) as it follows from Eq.(24) $g$ is linear in $\Delta_{0}^{2}$ if $\Delta_{0} \rightarrow 0$. The following function satisfies these conditions:

$$
g=\frac{\exp \left(-4 D_{p} \tau_{c r} / r_{m}^{2}+1\right)-1}{e-1}=\frac{\exp \left(\left(r_{m}^{2}-\Delta_{0}^{2}\right) / r_{m}^{2}\right)-1}{e-1}
$$

As it follows from the numerical comparison, both Eqs.(23) and (26) give similar results within a few percent accuracy even for trapped particles. . 


\section{FPPT code}

\subsection{Formulation}

For processes with a characteristic time greater than the $\alpha$-particle bounce period, which is $\tau_{b} \sim 10^{-6} \mathrm{sec}$ in TFTR, the distribution function of $\alpha$-particles can be represented as a function of particle integrals of motion $f_{\alpha}=f_{\alpha}\left(\mu, P_{\varphi}, p, t\right)$. The FPPT code solves the drift orbit averaged Fokker-Planck equation [12]

$$
\frac{\partial f_{\alpha}^{F}}{\partial t}=\left\langle S t\left(f_{\alpha}\right)\right\rangle+\left\langle S_{\alpha}\right\rangle-\frac{f_{\alpha}^{F}}{\tau_{\delta}}-\frac{f_{\alpha}^{F}}{\tau_{\text {conf }}} .
$$

Here angle brackets denote time averaging over the drift $\alpha$-particle orbit, $f_{\alpha}^{F}$ is the FPPT calculated $\alpha$-particle distribution function expressed in terms of variables $f_{\alpha}^{F}=$ $f_{\alpha}^{F}\left(v, P_{\varphi}, p, t\right)$, so that the distribution function introduced in the previous section is $f_{\alpha}\left(\mu, P_{\varphi}, p, t\right)=f_{\alpha}^{F}\left(v(\mu, p), P_{\varphi}, p, t\right), v(\mu, p)=\sqrt{2 B_{0} R_{0} \mu / p}$, with

$$
S_{\alpha}=S_{T R}(r, t) \exp \left(-\left(v-v_{\alpha 0}\right)^{2} / v_{T}^{2}\right) / \sqrt{\pi} v_{\alpha 0}^{2} v_{T}
$$

being the $\alpha$-particle source with $S_{T R}(r, t)$ taken from TRANSP analyzing code [13], and $v_{T}$ being the Doppler broadening, which was taken for a Maxwellian plasma with the temperature $T_{\text {eff }}=30 \mathrm{keV}$. The value of $T_{\text {eff }}=30 \mathrm{keV}$ for the broadening is obtained from measurements and changes by $\leq 10 \%$ during the discharge [14]. The collisional integral $S t\left(f_{\alpha}\right)$ in Eq.(28) includes only slowing down of alphas without scattering and velocity diffusion:

$$
S t\left(f_{\alpha}^{F}\right)=\frac{1}{v^{2} \tau_{s}} \frac{\partial\left(v^{3}+v_{*}^{3}\right) f_{\alpha}^{F}}{\partial v}+\frac{P_{\varphi}-e_{\alpha} \psi / 2 \pi m_{\alpha} c}{\tau_{s}}\left(1+v_{*}^{3} / v^{3}\right) \frac{\partial f_{\alpha}^{F}}{\partial P_{\varphi}}
$$

where $\tau_{s}$ is the slowing down time. The third term on the right hand side in Eq.(28) accounts for the magnetic ripple effect with $\tau_{\delta}$ being the $\alpha$-particle confinement time in the presence of toroidal ripple, which we determine based on the results of Ref.[15], where the approximate formula describing the smooth transition to the stochastic regime was obtained:

$$
\tau_{\delta}=\tau_{b} \frac{(r-a)^{2}}{\Delta r^{2}}=\tau_{b} \frac{(r-a)^{2}}{\rho_{L}^{2}} \frac{2 \epsilon^{3} \sin \theta_{b}}{\pi q^{3} N \delta^{2}\left(r_{b}, \theta_{b}\right)}\left(1+e^{6.9-5.5 \alpha}\right)
$$


where

$$
\alpha=\left(\frac{16 \pi N^{3} q^{5} R^{3}}{m_{\alpha} \omega_{c}^{2} r^{5}}\right)^{1 / 2} \mathcal{E}^{1 / 2} \delta\left(r_{b}, \theta_{b}\right) \frac{\theta_{b}(r / q)(d q / d r)+\cot \theta_{b}}{\sqrt{\sin \theta_{b}}}
$$

$\Delta r$ is the banana tip displacement for one bounce period, $r_{b}, \theta_{b}$ are the radial and poloidal coordinates of the banana tip, $\rho_{L}=v_{\alpha} / \omega_{c}$ is the Larmor radius, $\delta$ is the magnetic field ripple amplitude, which in TFTR is given by the expression:

$$
\delta(r, \theta)=\delta_{0} \exp \left[\left(\left(R_{0}+r \cos \theta-R_{r i p}\right)^{2}+b_{r i p}(r \sin \theta)^{2}\right)^{1 / 2} / w_{r i p}\right]
$$

$R_{0}$ is the major radius, $R_{\text {rip }}=2.25 \mathrm{~m}, b_{\text {rip }}=1.31, w_{\text {rip }}=0.1657 \mathrm{~m}, \delta_{0}=0.6 \times 10^{-5}$, $N=20$. Note that for deeply trapped $\alpha$-particles, $\sin \theta_{b} \simeq\left(v_{\|} / v\right) \sqrt{2 / \epsilon}$. For details see Ref.[15]. We note that the $\tau$ approximation for the ripple effect gives a smooth boundary between the loss region (near the plasma periphery) and the confined region (near the axis). The width of this transition region depends on plasma parameters and is about $10-20 \mathrm{~cm}$.

The last term in Eq.(28) is introduced to simulate the effect of finite confinement time on $\alpha$-particle distribution function in comparison of FPPT with experiment and will be studied later.

Eq.(28) was solved numerically by the method of integration over the particle charac-

teristics [16]. Direct guiding center orbit averaging was performed using TRANSP equilibrium along the drift orbit of $\alpha$-particle determined by three integrals $p, P_{\varphi}, v$. Test calculations to be shown later in the paper agree with TRANSP Monte Carlo simulations.

FPPT code is routinely used now for modeling of $\alpha$-particle distribution function in TFTR.

\subsection{Comparison of FPPT and TRANSP Monte-Carlo calculations}

FPPT calculated spectra of $\alpha$-particles are compared with TRANSP Monte-Carlo results [13] in Fig. 2 for shot \#86644 at $4.15 \mathrm{sec}$, i.e. $0.15 \mathrm{sec}$ after the Neutral Beam Injection (NBI) was turned off. To eliminate the statistical error in Monte-Carlo simulations we present the energy distribution averaged over the pitch angle. TRANSP 
uses a delta function for the energy dependence of fusion product source, which explains different behavior of distributions at $\mathcal{E}_{\alpha}>3 \mathrm{MeV}$. Another domain where both codes differs is $\mathcal{E}_{\alpha}<0.5 \mathrm{MeV}$. This is because FPPT is based on the method of integration over the characteristics, which does not allow inclusion of the second derivative operator into the code. Thus, FPPT does not have pitch angle scattering in the collisional operator, which results in the radial transport in TRANSP. This effect should be taken into account while comparing with experiments.

Figure 3 presents a comparison of energetic $\left(\mathcal{E}_{\alpha}>1 \mathrm{MeV}\right) \alpha$-particle profiles for the same shot as in Fig. 2. Fig. 3 shows the density profiles (a) and stochastic ripple loss profiles (b) integrated in energy and pitch angle. The TRANSP model of ripple loss implies instant loss if the banana tip of fast particle is in the loss region. Therefore the boundary between the stochastic and nonstochastic regions is sharp. FPPT uses the model formula Eq. (31) for the confinement time in rippled magnetic field, which from the figure: (1) gives the same boundary for the stochastic region, and (2) gives the similar integral losses. The model based on Eq. (31) has some finite width of the transition to the stochastic region, which determines the accuracy of our tau approximation. More on the ripple effects on alphas can be found in Ref.[17].

\subsection{FPPT simulations for the pellet charge exchange diagnostic}

The PCX diagnostic was designed and installed on TFTR to measure the well trapped confined fast particle distributions in D-T plasmas $[18,19]$. A neutral particle mass and energy analyzer is used to detect fast particles converted to neutrals in the ablation cloud of the injected impurity pellet. The neutral particle analyzer is located in the tokamak midplane and allows measurements of the local density and energy spectra of fast particles during the pellet penetration into the plasma. At the moment of neutralization, the fast particle has a pitch angle $\chi=v_{\|} / v=-0.048$ in the midplane, which corresponds to the inner leg of its drift banana orbit. PCX was used to measure the distribution of fusion $\alpha$-particles in DT TFTR experiments and effects of magnetic field ripple and sawtooth oscillations on energy spectra and radial profiles $[1,2,17,20]$.

Figure 4 represents the FPPT calculations of the evolution of $\alpha$-particle slowing 
down energy distribution function taken at the plasma center and at the PCX measured pitch angle $\chi=-0.048$. Calculations were done for discharge \#86291 with NBI power $P_{b}=15 \mathrm{MW}$, except one curve at $0.012 \mathrm{sec}$ for discharge \#86299 with $P_{b}=20 \mathrm{MW}$. PCX data [20] are also shown. In the shot \#86291 the measurements were done at 0.2 sec after 1 sec NBI, while in the shot \#86299 the energy spectrum was measured in a "beam blip" case at $0.02 \mathrm{sec}$ after a beam pulse of $0.1 \mathrm{sec}$ duration. The typical alpha slowing down time in TFTR is $\tau_{s} \sim 0.5 \mathrm{sec}$, which is much larger than the "blip" duration and, therefore, can yield information about the energy distribution of $\alpha$-particles. The modeling of FPPT shows reasonable agreement with PCX data. As we mentioned above, FPPT may not be good for the low energy part of alphas distribution function as it does not include pitch angle scattering. This may explain higher data point for the two lowest energy channels with good statistics in discharge \#86291.

Figure 5 shows the sensitivity of the modeling to the confinement time $\tau_{\text {conf }}$ introduced in Eq.(28). The confinement time was taken constant during the discharge and not to be a function of particle energy. The curves deviate from the curve $\tau_{\text {conf }}=\infty$ at lower energy, because the longer a particle lives in the plasma the more probable it will be lost. The confinement time for this case was $\tau_{s} \simeq 0.3 \mathrm{sec}$. The best fit corresponds to the longest confinement time (at least $\tau_{\text {conf }}>3 \tau_{s}$ ), which supports the idea that the slowing down is classical without additional loss machanisms. Fig. 5 also shows the sensitivity of PCX measured spectra to additional losses.

As we mentioned above, FPPT can not treat the diffusion self consistently. To model diffusion and to see the sensitivity of the method to radial diffusion we used the formula Eq.(23) for diffusion in $P_{\varphi}$ and $p$ (with the substitution $P_{\varphi} \rightarrow p$ for $P_{\varphi}$ diffusion). The formula Eq.(23) was used once, right before the time of PCX mesurements. The value $\Delta_{0}$ was taken as a function of particle energy and diffusion coefficient $D_{P_{\varphi}, p}$ as follows

$$
\Delta_{0}=\sqrt{D_{P_{\varphi}, p} \frac{\tau_{s}}{2} \ln \frac{\mathcal{E}_{\alpha}}{\mathcal{E}_{\alpha 0}}} .
$$

Results show that $D_{P_{\varphi}}$ does not change the profiles of alphas, but affects the spectra (we can write $\left.\tau_{\text {conf }} \simeq a^{2} / D_{P_{\varphi}}\right)$. In contrast, $D_{p}$ has effect on the profiles which is illustrated in Figure 6. It shows the FPPT simulation of $\alpha$-particle profiles at different values of $D_{p}$ 
and fixed particle energy $\mathcal{E}_{\alpha}=1.21 \mathrm{MeV}$. We can see that the best fit to experimental PCX profiles [2] is at the lowest diffusion or $D_{p}<0.01 \mathrm{~m}^{2} \mathrm{sec}^{-1}$. We again conclude that there are, no significant anomalous processes in slowing down in terms of energy diffusion.

\section{Simulation and observation of confined $\alpha$-particle sawtooth redistribution}

Figure 7 presents an example of the PCX alpha density profile for discharges \#84549 and \#84550 with and without sawtooth activity, respectively, for a given energy $\mathcal{E}=$ $0.82 \mathrm{MeV}$. Alpha particles appear farther out from the plasma center in the discharge with a sawtooth. This behavior is typical for most discharges with sawteeth [2].

\subsection{FPPT trapped $\alpha$-particle simulations}

Figure 7 also presents the results of the FPPT calculations of $\alpha$-particle profiles for the sawtooth free discharge \#84550. Numerical results are normalized to the PCX data at $R=2.65 \mathrm{~m}$. Keeping the same normalization, we present also calculated profiles for the discharge \#84549 neglecting the sawtooth effect. The profile is similar to the calculations for the discharge \#84550. Any mixing without changing the energy of trapped particles will lead to a drop of the central $\alpha$-particle density value as it is illustrated on Fig. 7 for the flat post crash alphas distribution in $P_{\varphi}$.

Figure 8 shows the PCX measured spectra for the same discharges but at different major radius: $R=2.65 m$ for $\# 84550$ and $R=2.9 m$ for \#84549. Also shown are FPPT calculated $\alpha$-particle spectra for the sawtooth free discharge \#84550 where we performed the normalization, and calculated spectra for the \#84549 discharge without and with mixing using different critical energy $\mathcal{E}_{c r}$. In numerical analysis we used the mixing formula from Eq.(23). The best adjusted value for $\mathcal{E}_{c r}$ is obtained from the comparison with other spectra and equals $\mathcal{E}_{\text {cr }} \simeq \mathcal{E}_{\alpha 0}$. Note that the time of PCX measurements is $120 \mathrm{msec}$ after the crash. This is comparable with the slowing down time, which means that PCX analyzes particles which at the crash had energy equal to the birth energy of 
$\alpha$-particle.

The calculated profiles are compared with measurements in Figure 9. In all the comparisons, the PCX signal is assumed to be proportional to the density of $\alpha$-particles, which is true if the density of the cloud is more than certain critical value [18]. This assumption allows us to compare the $\alpha$-particle profiles for two different discharges. One can see from profile comparisons (Fig.9) that again $\mathcal{E}_{c r} \geq \mathcal{E}_{\alpha 0}$ gives the best fit to the data.

In all the calculations above we used the mixing radius $r_{m}=1.5 r_{s}\left(q\left(r_{s}\right)=1\right)$, which determines the mixing region. Mixing radius is the second critical parameter of the model. Its value was also chosen to give the best fit to the data.

Within the accuracy of the measurements and the model, good agreement is seen in a comparison of the PCX profile and spectra measurements with FPPT model.

\subsection{FPPT passing $\alpha$-particle simulations}

In Figure 10 we present the profiles of passing particles calculated at the pitch angle $\chi=0.8$ in the midplane and compare it with the trapped particle results at $\chi=-0.048$. This figure shows that the mixing radius for both group of particles is the same, even though the physical reasons of the mixing are different. Trapped particles are redistributed because they change energy in the perpendicular electric field, while passing particles change only $P_{\varphi}$ in the perturbed magnetic fields.

\section{Summary}

We propose a model for the fusion $\alpha$-particle mixing during the sawtooth crash. The model is based on the $\mu$ conservation and an energy change in a helical perturbed perpendicular electric field. The model gives good agreement with PCX measurements of the trapped $\alpha$-particle distributions in DT TFTR plasmas. It describes the spectra and the broadening of alpha density profiles after the crash. The FPPT sawtooth model can be applied to both passing and trapped particles. However, trapped particles are more affected by the electric field than passing particles, which allows the energy redistribution 
(using trapped particle diagnostic $\mathrm{PCX}$ ) and the $P_{\varphi}$ (minor radius) redistribution (using mostly passing particle diagnostic $\alpha$-CHERS) to be studied separately.

FPPT model has two critical parameters, which are the critical energy $\mathcal{E}_{c r}$ and the mixing radius $r_{m}$. Comparisons with the experimental data for one DT TFTR discharge \#84549 gives $\mathcal{E}_{\text {cr }} \simeq \mathcal{E}_{\alpha 0}$. Such particles slowed down to $\mathcal{E} \simeq 1-2 \mathrm{MeV}$ by the time (120 msec) of PCX measurements. The predicted $\mathcal{E}_{c r} \simeq \mathcal{E}_{\alpha 0}$ gives $\tau_{c r} \simeq 10 \mu \mathrm{sec}$ for discharge \#84549, which is in reasonable agreement with sawtooth measurements [5]. The second critical parameter $r_{m}=1.5 r_{s}, q\left(r_{s}\right)=1$ is equal (within the accuracy of the model) to the Kadomtsev model for parabolic plasma current profile $r_{m}=\sqrt{2} r_{s}$ [11]. The model can be used to study the passing particle mixing, but more realistic $P_{\varphi}$ redistributions must be included.

Also more detailed measurements of the sawtooth crash (e.g. $n_{e}, T_{e}, \tau_{c r}$ etc.) for comparison with the model should be obtained.

The model presented here and the FPPT code can be used to examine the implications for $\alpha$-particles in ITER.

\section{ACKNOWLEDGMENTS}

We would like to thank Drs. S. V. Putvinskii, B. Stratton, R. White, Ye Zhao, L. E. Zakharov, S. Zweben for useful discussions. 


\section{References}

[1] FISHER, R. K., et.al., Phys. Rev. Lett. 75 (1995) 846.

[2] PETROV, M. P., et.al. Nucl. Fusion 35 (1995).

[3] STRATTON, B. C., et.al. submitted to Nucl. Fusion.

[4] KOLESNICHENKO, YA. I., YAKOVENKO, Yu. V., Nucl. Fusion 32 (1992) 449.

[5] McGUIRE, K., et. al. Phys. Fluids 2 (1990) 1287.

[6] LICHTENBERG, A. J., Nucl. Fusion 24 (1984) 1277.

[7] ZHAO, YE, WHITE, R., private communication.

[8] ANDERSON, D., et. al., Nuclear Fusion 34 (1994) 217.

[9] World Survey of Activities in Controlled Fusion Research [Nuclear Fusion special supplement 1991],(International Atomic Energy Agency, Vienna, 1991)]

[10] KOLESNICHENKO, Ya. I., YAKOVENKO, Yu. V., Phys. Scripta 45 (1992) 133.

[11] KADOMTSEV, B. B., Fiz. Plasmy 1 (1975) 710; Sov. J. Plasma Phys. 1 (1976) 389.

[12] PUTVINSKIJ, S. V. , Reviews of Plasma Phys. V.18.

[13] BUDNY, R. V., Nucl. Fusion 32 (1992) 429.

[14] KRASILNIKOV, A. V., et. al., Bull. Amer. Phys. Soc. 40 (1995) 1768.

[15] YUSHMANOV, P. N., Nucl. Fusion 23 (1983) 1599.

[16] GORELENKOV, N. N., PUTVINSKI, S. V., Sov. J. Plasma Phys., 15 (1989) 145.

[17] DUONG, H. H., et.al., PPPL Report \# 3178, submitted to Nuclear Fusion.

[18] FISHER, R. K., et.al., Rev. Sci. Instrum. 63 (1992) 4499.

[19] MEDLEY, S. S., et.al., in Proceedings of 20th European Conference on Controlled Fusion and Plasma Physics, Lisbon, 1993, (European Physical Society, Petit-Lancy, Switzerland, 1993), Part III, p.1183.

[20] MEDLEY, S. S., et.al., PPPL Report \#3173, submitted to Rev. Sci. Instrum. 


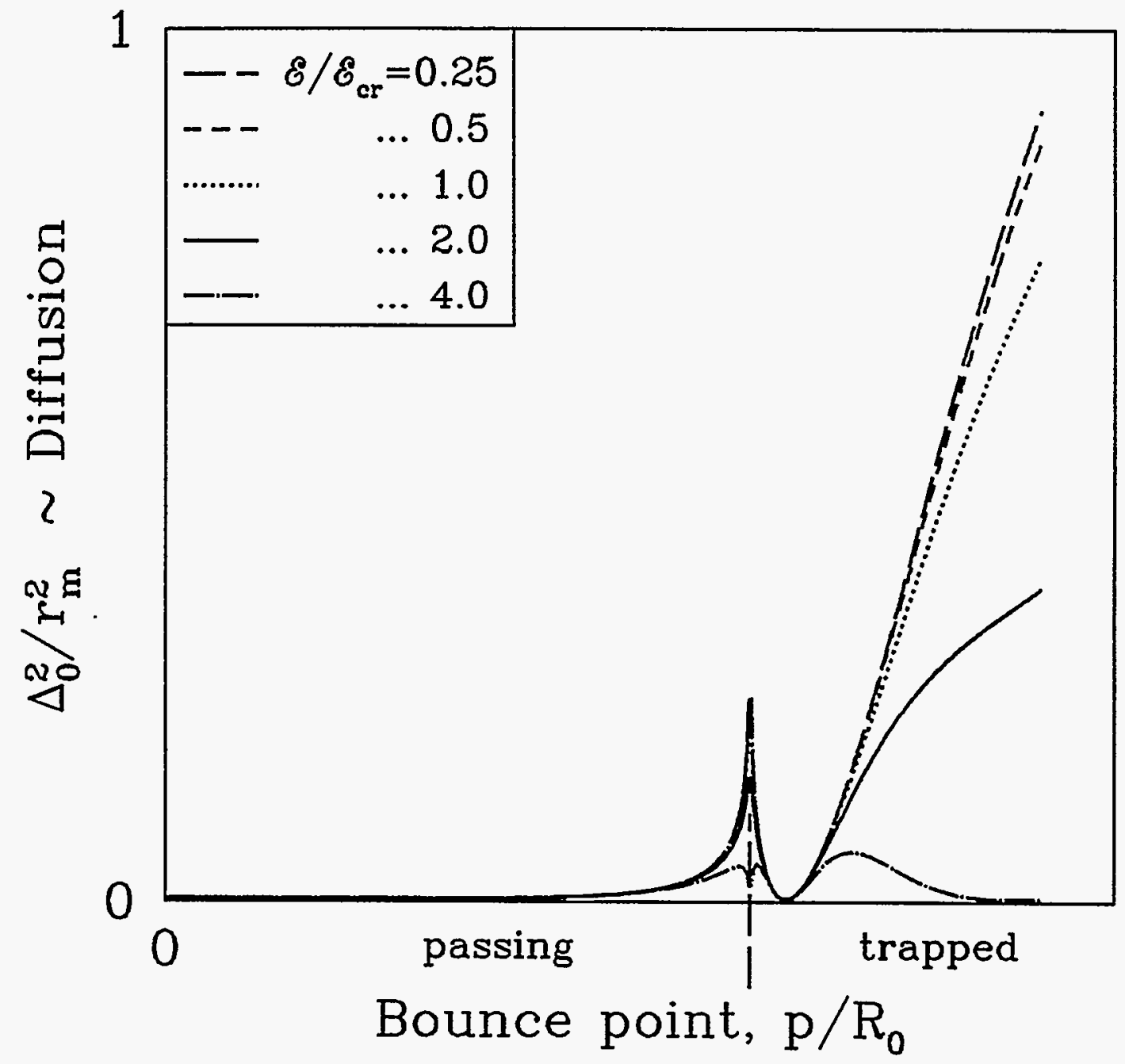

Figure 1: Fast particle bounce point displacement $\Delta_{0}^{2} / r_{m}^{2}$ (see Eq.(21)) versus the position of the bounce point $p / R_{0}$ at $\epsilon=.2, \mathcal{E} / \mathcal{E}_{\text {cr }}=1 / 4,1 / 2,1,2,4$, respectively. 


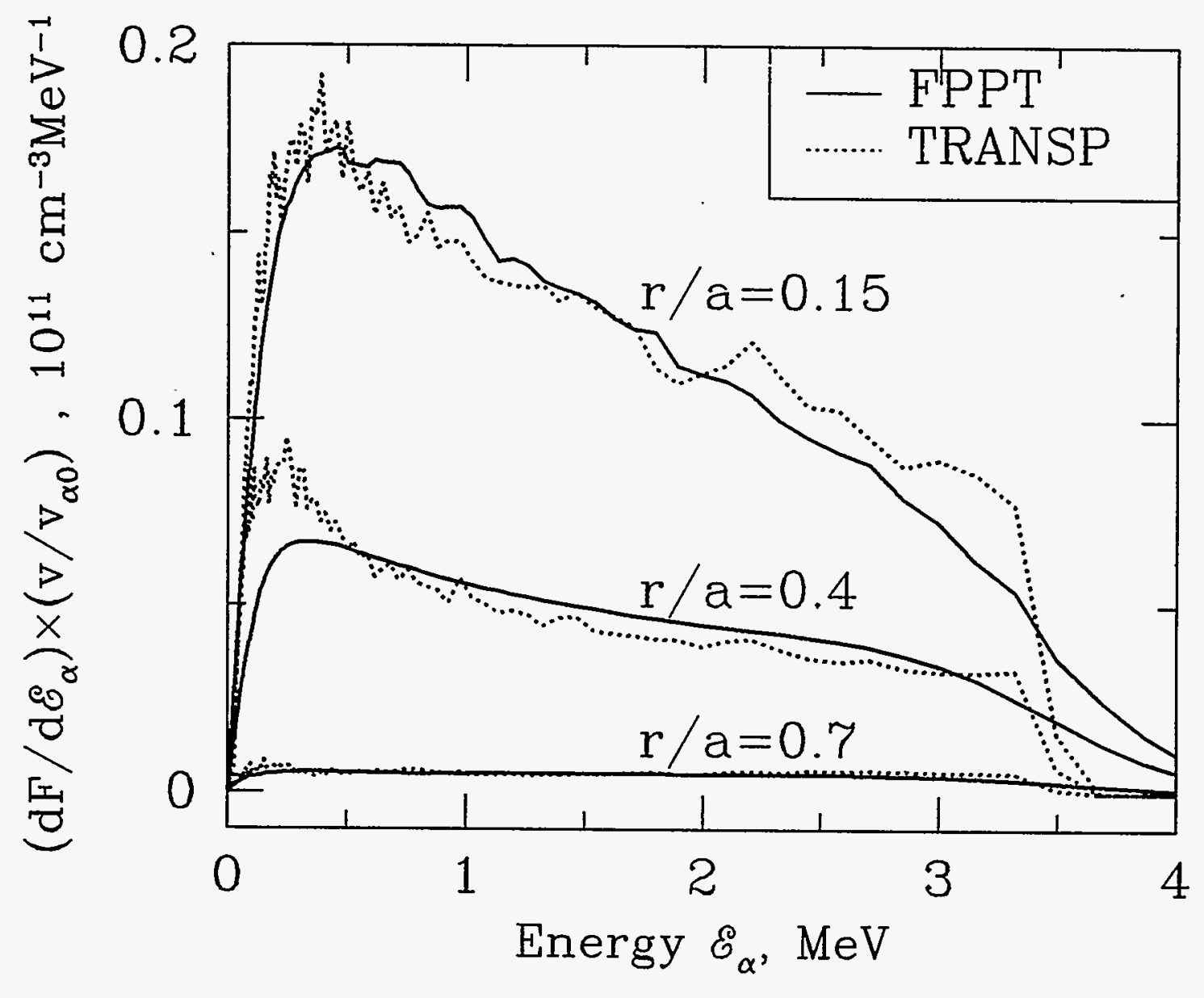

Figure 2: Comparison of FPPT and TRANSP Monte-Carlo calculations of $\alpha$-particle distribution functions versus energy. Spectra are averaged over the pitch angle and taken at different plasma minor radius in the equatorial plane at the low magnetic field side. The results are for TFTR shot \#86644 at $4.15 \mathrm{sec}$, i.e. $0.15 \mathrm{sec}$ after NBI. 


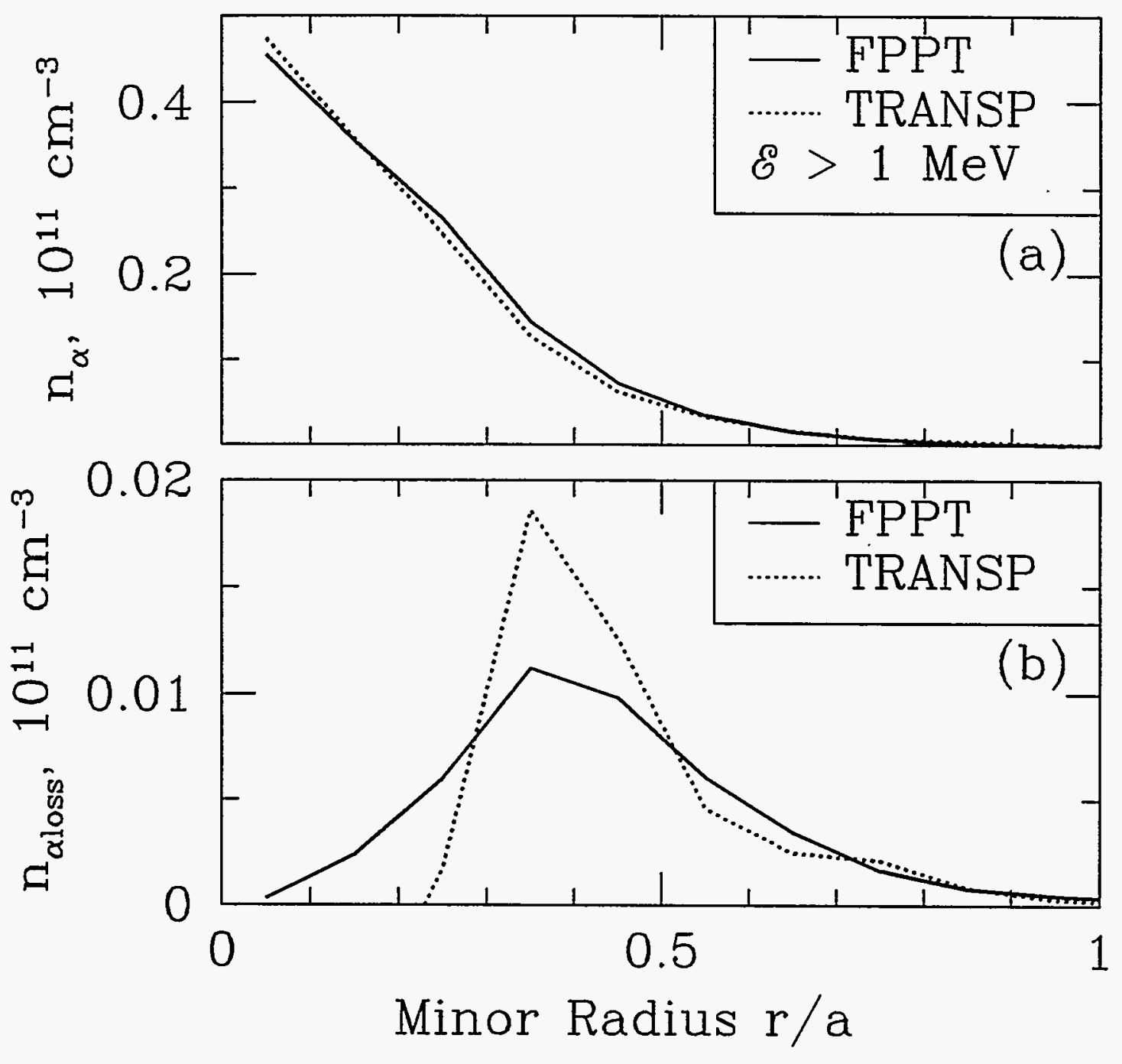

Figure 3: FPPT and TRANSP calculated energetic $\left(\mathcal{E}_{\alpha}>1 \mathrm{MeV}\right) \dot{\alpha}$-particle profiles of the (a) density and (b) stochastic loss due to the magnetic field ripples for the same shot as in the Fig. 2. 


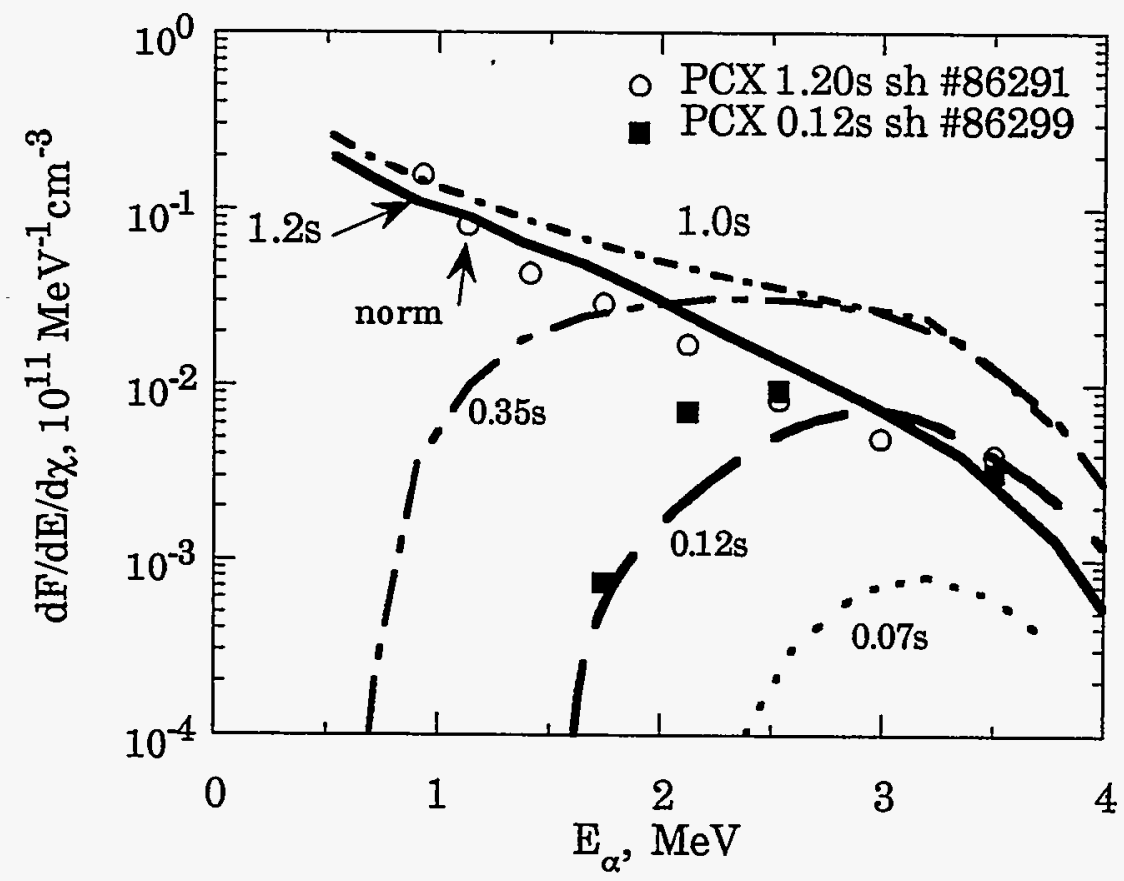

Figure 4: FPPT calculation of the evolution of $\alpha$-particle ditribution function in TFTR. Curves correspond to the shot \#86291 except curve at $0.12 \mathrm{sec}$, which is plotted for shot \#86299. Shown also are the PCX data for these shots [20]. 


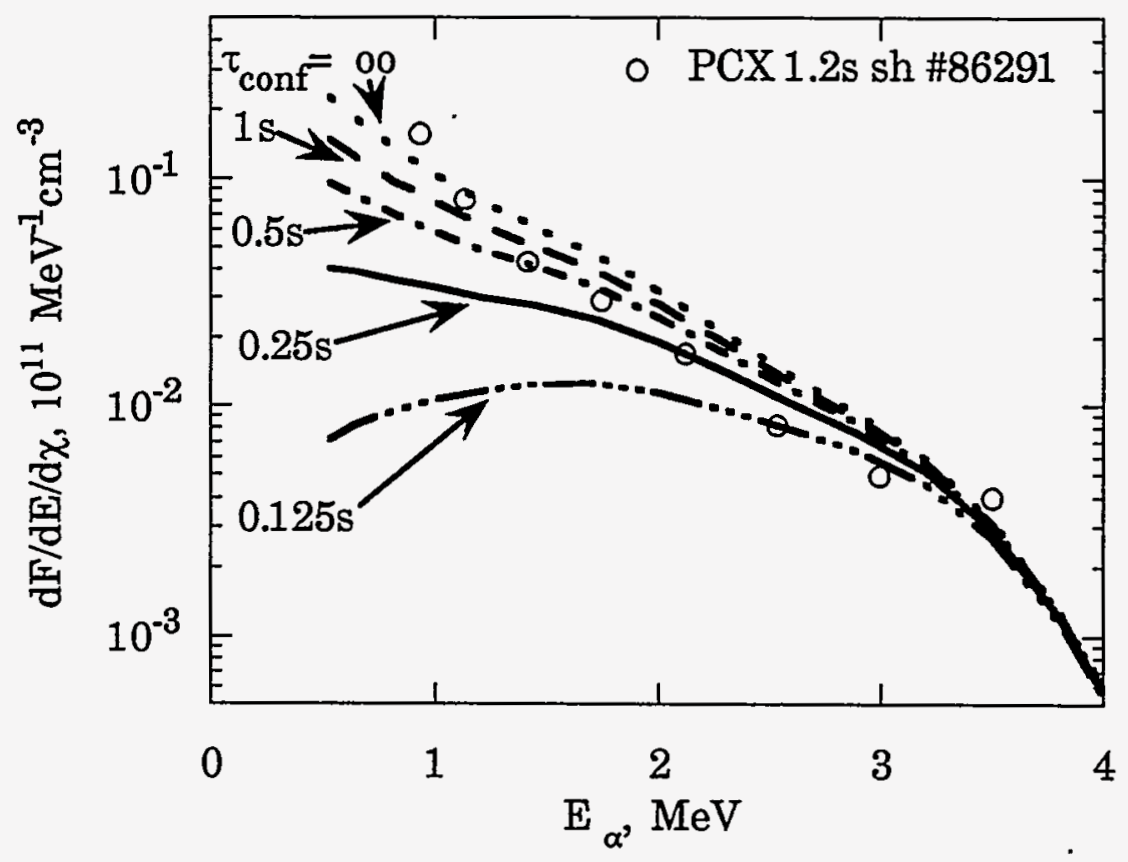

Figure 5: Comparison of PCX measurements with FPPT calculated spectra with different confinement times. 


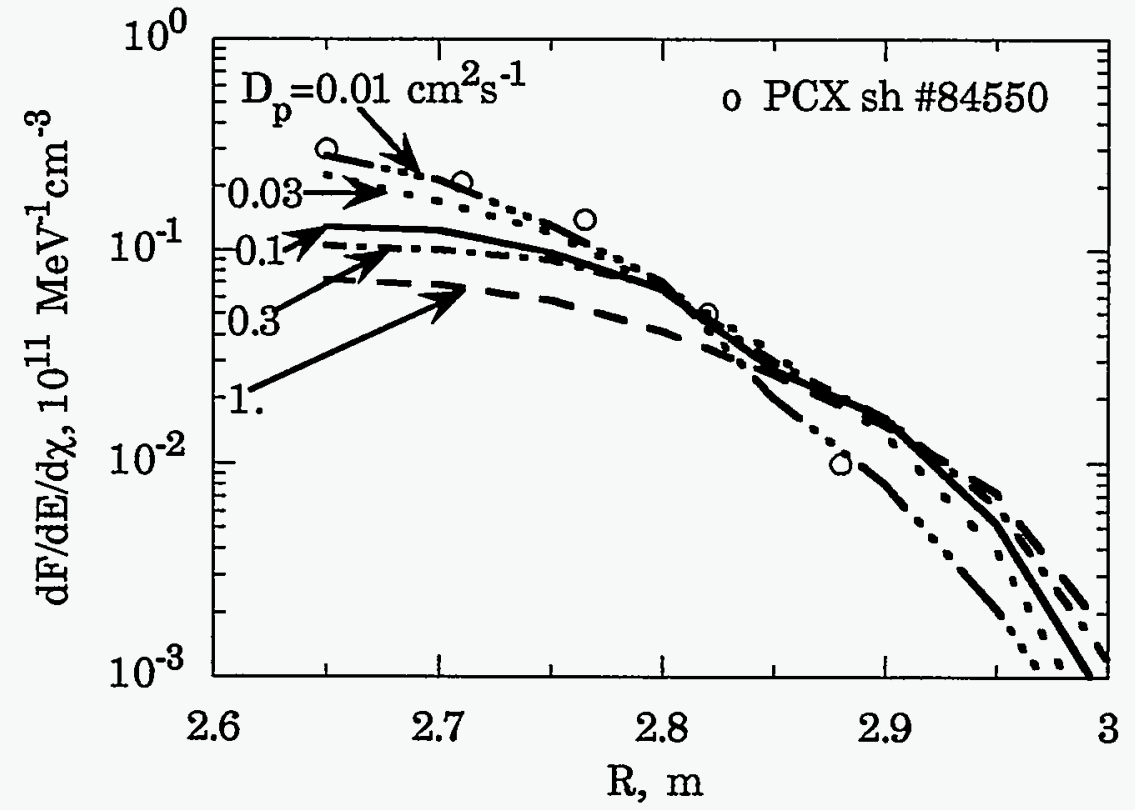

Figure 6: Simulation of $\alpha$-particle distribution function profiles for different diffusion coefficients of the banana tip $p$ diffusion. PCX measured data are also shown. 


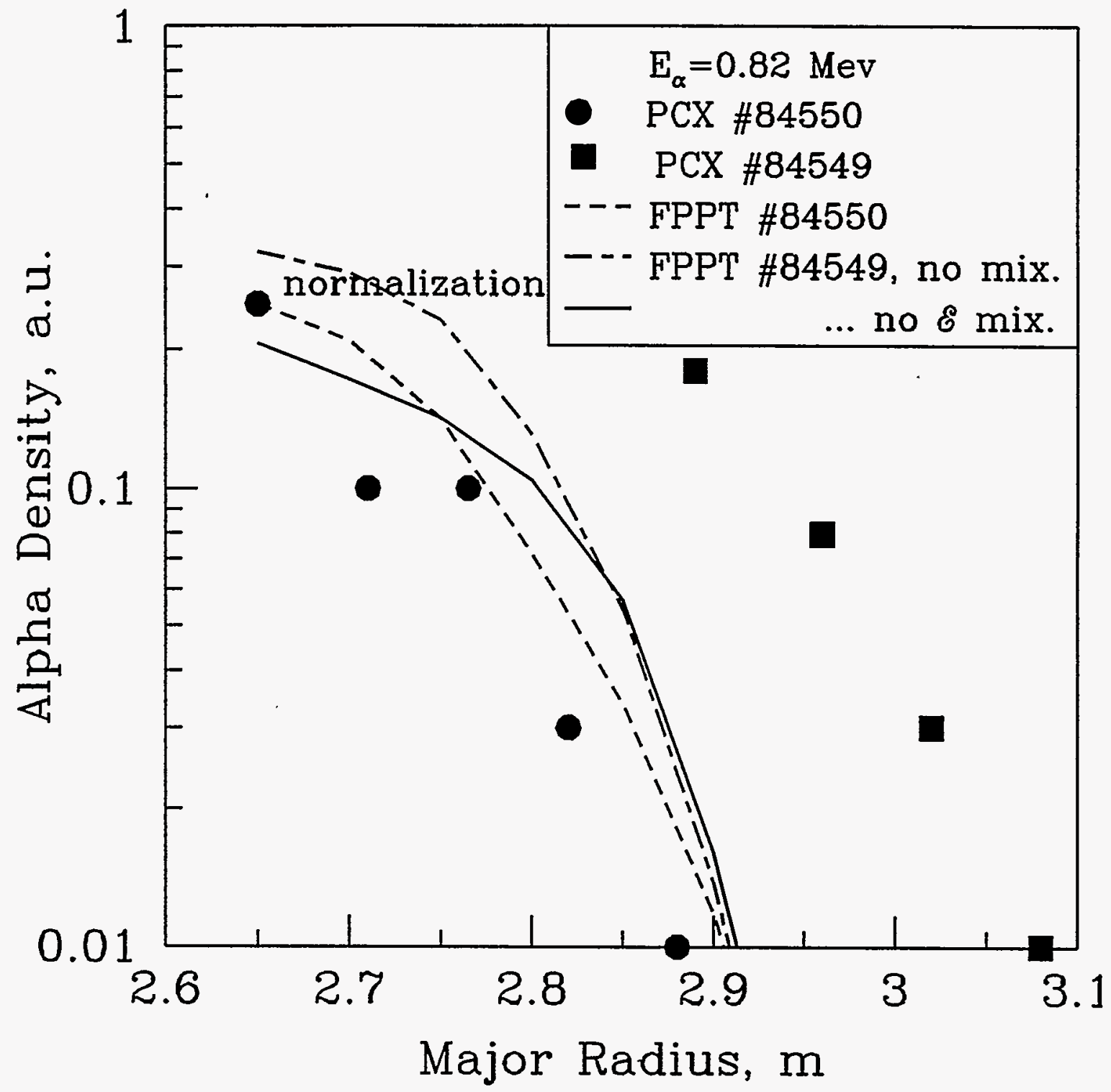

Figure 7: Alpha particle radial density profiles for $\mathcal{E}_{\alpha}=0.82 \mathrm{MeV}$ from PCX data for a sawtooth free discharge \# 84550 and a sawtooth discharge \# 84549. Shown also are the results of FPPT modeling. 




Figure 8: PCX alpha energy spectra for a sawtooth free discharge \#84550 (solid circles) at $R=2.65 \mathrm{~m}$ and a sawtooth discharge $\# 84549$ (open circles) at $R=2.9 \mathrm{~m}$. FPPT modeling is presented as a spectrum without mixing for the sawtooth free discharge \#84550 (curve 1) and for discharge \#84549 (curve 6) with sawtooth. The calculations with mixing are for parameters $\mathcal{E}_{c r} / \mathcal{E}_{\alpha 0}=2$. (curve 2), 1. (curve 3), 0.5 (curve 4), 0.25 (curve 5). 


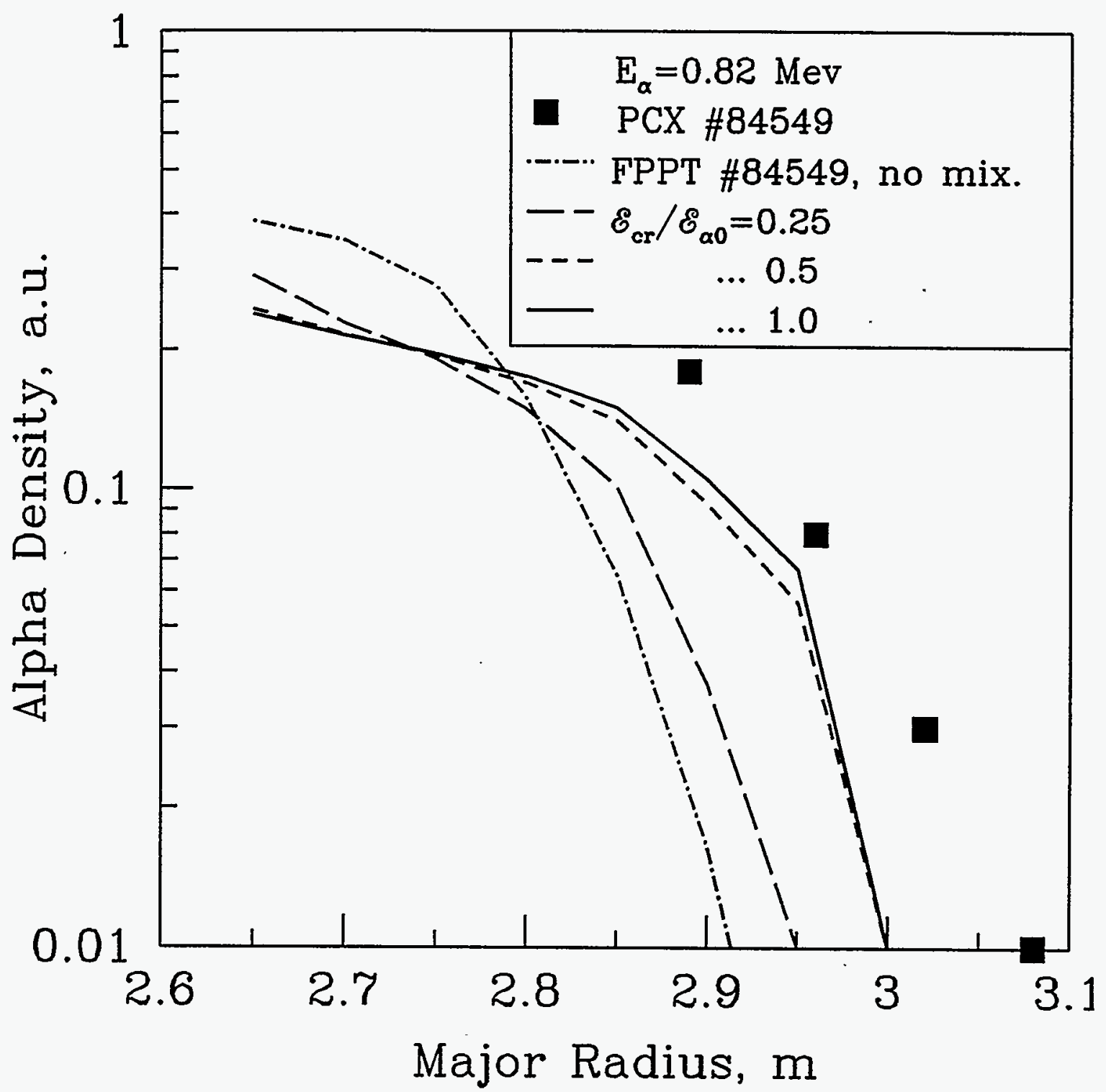

Figure 9: Alpha particle radial density profile for $\mathcal{E}_{\alpha}=0.82 \mathrm{MeV}$ from PCX data for the discharge with a sawtooth \#84549. Shown also are the results of FPPT modeling of trapped particle profiles for different critical energies. 


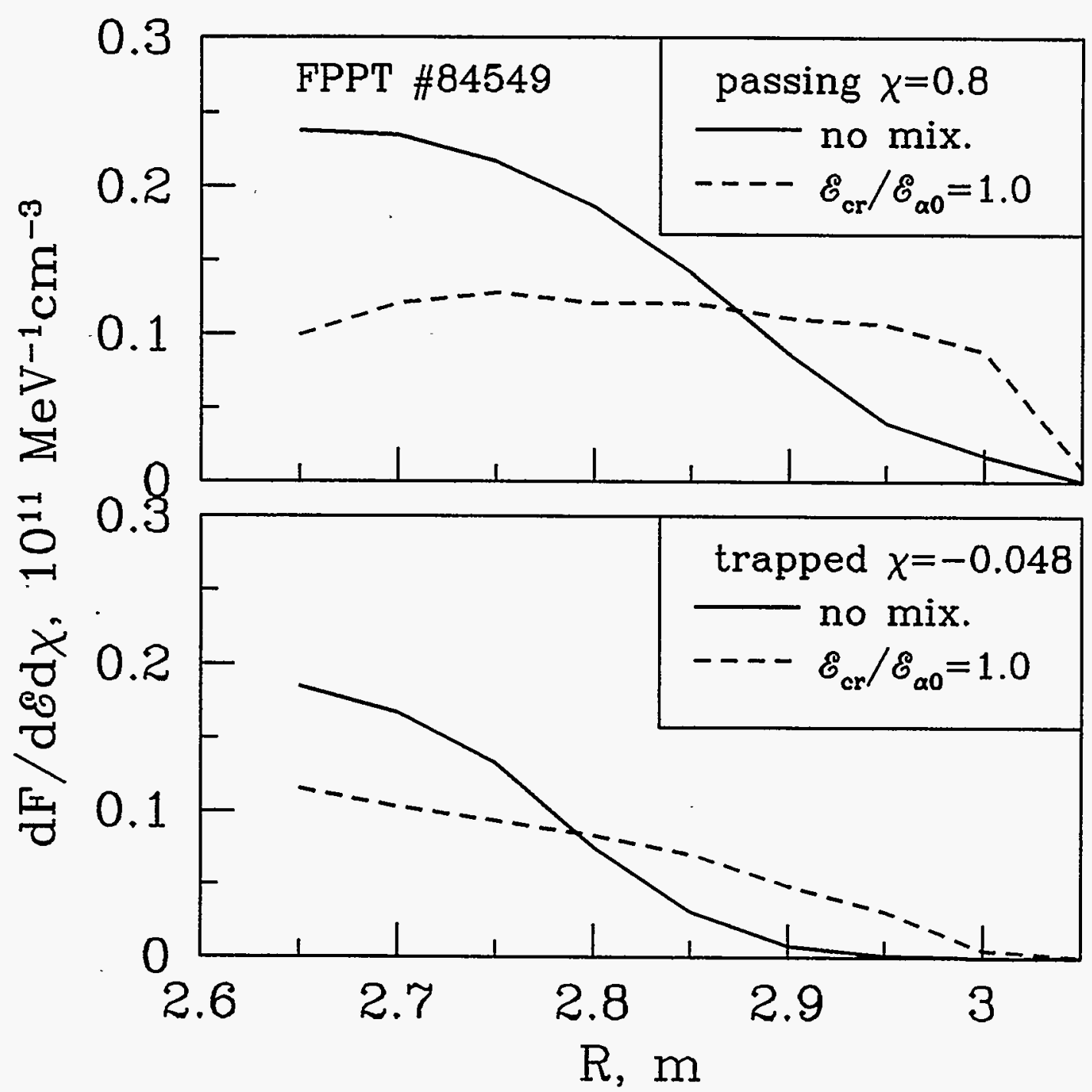

Figure 10: Passing (midplane pitch angle $\left.\chi_{\text {mid }}=0.8\right)$ and trapped $\left(\chi_{\text {mid }}=-0.048\right)$ $\alpha$-particle profiles for $\mathcal{E}_{\alpha}=0.82 \mathrm{MeV}$ from FPPT calculatoins for the discharge \#84549 with a sawtooth. 